\title{
A Time Difference of Arrival-based Localization Algorithm for Wireless Sensor Networks
}

\author{
https://doi.org/10.3991/ijoe.v12i11.6228 \\ Ji Songbo \\ Inner Mongolia University of Technology, Hohhot, China
}

\begin{abstract}
Aimed at solving the problem of local divergence and low data accuracy, this paper introduces a new Time Difference of Arrival(TDOA)-based localization algorithm (TBL) for the large-scale, high-density wireless sensor networks which are designed for real-time surveillance and unexpected incidents management. In particular, several means to improve the accuracy of distance measurement are investigated, and the TDOA method, based on the sound wave and electromagnetic wave to locate in the large-scale WSN, is discussed. Also, the well-designed circular location process has the advantage of better positioning accuracy and coverage percentage. Simulation results have confirmed the effectiveness of the formed TBL algorithm.
\end{abstract}

Index Terms-distributed data, TDOA-based localization algorithm, wireless sensor networks

\section{INTRODUCTION}

Ad hoc wireless sensor network has recently attracted much interest in the wireless research community as a fundamentally new tool for a wide range of monitoring and data-gathering applications. Environmental control and monitoring, smart rooms, robot control, inventory systems, and interactive virtual worlds are only a few examples. The underlying technology that drives the emergence of sensor applications is the rapid development in the integration of digital circuitry, which will bring us small, cheap, autonomous sensor nodes in the near future. New technology offers new opportunities, but it also introduces new problems. This is particularly true for sensor networks where the capabilities of individual nodes are very limited. Hence, cooperation between nodes is required, but energy conservation is a major concern, which implies that communication should be minimized. These conflicting objectives require outstanding solutions for many situations [1-3].

Measuring the physical positions of sensors is a fundamental and crucial problem in wireless ad hoc sensor network operation for several important reasons. We briefly list two of them in the following: first, in order to use the data collected by sensors, it is often necessary to have their position information stamped. For example, in order to detect and track objects with sensor networks, the physical position of each sensor should be known in advance for identifying the positions of detected objects. In addition, many communication protocols of sensor networks are built on the knowledge of the geographic positions of sensors. However, in most cases, sensors are deployed without their position information known in advance, and there is no supporting infrastructure available to locate them after deployment. It is necessary to find an alternative approach to identify the position of each sensor in wireless sensor networks after deployment.

Extensive research has been done on localization for wireless networks. A general survey is found in [4]. Global Positioning System (GPS) has been widely used for localization service [5-6]. Although it is possible to find the position of each sensor in a wireless sensor network with the aid of GPS installed in all sensors, it is not practical to use GPS due to its high power consumption, expensive price and line of sight conditions. The solutions developed in ubiquitous computing [4] are generally not applicable for ad hoc wireless sensor networks as they require too much processing power and energy.

Centralized localization methods depend on sensor nodes transmitting data to a central location, where computation is performed to measure the location of each node. Doherty developed a centralized technique using convex optimization to estimate positions based only on connectivity constraints given some nodes with known positions [7-8]. MDS-MAP [9] improves on these results by using a multidimensional scaling approach, but still requires centralized computation. Requiring central computation would be infeasible for ad hoc wireless sensor networks.

Distributed localization methods do not require centralized computation, and rely on each node determining its location with only limited communication with nearby nodes. These methods can be classified as range-based and range-free. Range-based methods use distance estimates or angle estimates in location calculations, while range-free methods depend only on the contents of received messages.

In this paper, we propose a TDOA-based localization scheme for wireless sensor networks and validate the scheme by simulation. This location measurement scheme avoids the drawbacks of many existing schemes for outdoor sensor location detection. Our simulations show that our scheme is potentially very effective.

\section{METHOD AND ALGORITHM}

The classic Chan algorithm could attain all TDOA by measuring, and obtain a specific analytical solution, however, the performance would be affected in NLOS environment. Taylor series expansion method could conduct recursive operation on the initial estimated location value of mobile station and continuously correct the estimated location value of mobile station to make it approach the real location value, however, this algorithm would significantly affected by the initial location, and the operation process is rather complicated. Therefore, we can explore some location algorithm to obtain one relatively precise initial location at first, then the obtained initial location 
can be used to conduct resolution with Taylor series expansion method and finally the location result which is more precise will be received.

The literature[3,4] presented a location algorithm which was based on Fang algorithm and Taylor series expansion, it eliminated the location estimation which had eliminated the estimated location value which deviated excessively to improve the precision of location. The literature proposed another synergy location algorithm, it conducted weighted calculation of the results attained by different location algorithm, and the estimated value which had been treated was used as the location coordinates of mobile station. According to the thoughts in literature, we present a modified location algorithm which is based on Chan algorithm and Taylor series expansion.

Assumed that the base station of community where the mobile station is located is noted as No.1, and meanwhile it act as the serving base station for the location system. If the measured TOA of $\mathrm{N}_{\mathrm{BS}}$ base stations has been attained, these TOA values are subtracted from the measured TOA values of serving base station and $\left(\mathrm{N}_{\mathrm{BS}}-1\right)$ TDOA values would be got. If data of the involved $m(m \leqslant n-1)$ base stations is always used during each location, $m$ TDOA values can be randomly chosen from these $\left(\mathrm{N}_{\mathrm{BS}}-1\right)$ ones to become one group and it has $C_{N_{B S-1}}^{m}$ kinds of possibilities.

Chan algorithm is used to obtain the initial estimated location value of mobile station under $C_{N_{B S-1}}^{m}$ kinds of conditions respectively. It is noted as $\left(\hat{x}_{k}, \hat{y}_{k}\right)$, besides, $\mathrm{k}=$ $1,2, \cdots, C_{N_{B S-1}}^{m}$. With the increasing distance between mobile station and base station error of the estimated $\left(\hat{x}_{k}, \hat{y}_{k}\right)$ also increase, which would significantly affect the ultimate estimated location result of mobile station, therefore, the initial estimated values which deviates excessively should be eliminated according to some disciplines. $\left(\hat{x}_{k}, \hat{y}_{k}\right)$ should be eliminated when the following condition was satisfied:

$$
\left|\sqrt{\left(x_{1}-\hat{x}_{k}\right)^{2}+\left(y_{1}-\hat{y}_{k}\right)^{2}}-R_{1}\right| \leq R_{\text {threshold }}
$$

Where, $R_{\text {threshold }}$ is one threshold, which could be alternative to control the error of $\left(\hat{x}_{k}, \hat{y}_{k}\right)$ as required.

Assumed that estimated values of $\eta$ initial coordinates remained after eliminating the values which deviates excessively, the values of the $\eta$ coordinates are averaged and got:

$$
\left[\begin{array}{l}
x_{0} \\
y_{0}
\end{array}\right]=\left[\begin{array}{l}
\frac{1}{\eta}\left(\sum_{k=1}^{\eta} \hat{x}_{k}\right) \\
\frac{1}{\eta}\left(\sum_{k=1}^{\eta} \hat{y}_{k}\right)
\end{array}\right]
$$

$\left(x_{0}, y_{0}\right)$ is the initial estimated location value of mobile stations which is attained with Chan algorithm. $\left(x_{0}, y_{0}\right)$ is substituted into Taylor series expansion method to conduct recursive resolution and one location result could be attained.

The location results attain with Chan algorithm and Taylor series expansion method should be weighted. The residual between the measured value and location result is defined in the literature [4]:

$$
R_{e s}=\sum_{i=1}^{N B S}\left(R_{i}-\hat{R}_{i}\right)^{2}
$$

Where, $\hat{R}_{i}=\sqrt{\left(\hat{x}-x_{i}\right)^{2}+\left(\hat{y}-y_{i}\right)^{2}},(\hat{x}, \hat{y})$ is the obtained location coordinates of mobile station with location algorithm, $\left(x_{k}, y_{k}\right)$ is the coordinates of No.i base station involved in location, $R_{i}$ is the obtained distance between the mobile station and No.i base station according to TOA value, $N_{B S}$ is the amount of base stations involved in location. When some location algorithm is adopted, if $R_{e s}$ is relatively small, it demonstrates that the obtained coordinate location of the mobile station using this algorithm is more precise; if $R_{e s}$ is relatively large, it indicates that errors of the obtained results using this algorithm is relatively large. The algorithm which is more precise should make up larger proportion in ultimate weighting. The calculation formula of weighing coefficient $R_{k}$ is given in literature. If $\mathrm{k}$ kinds of location algorithms are used to conduct operation on the same group of TDOA, respectively, weighting coefficient $R_{k}$ of No.k location algorithm could be expressed as:

$$
\begin{aligned}
R_{k} & =\frac{R_{e s}^{k}}{N_{B S}}=\frac{1}{N_{B S}} \sum_{i=1}^{N_{B S}}\left(R_{i}-\hat{R}_{i}^{k}\right)^{2} \\
& =\frac{1}{N_{B S}} \sum_{i=1}^{N_{B S}}\left(R_{i}-\sqrt{\left(\hat{x}^{k}-x_{i}\right)^{2}+\left(\hat{y}^{k}-y_{i}\right)^{2}}\right)^{2}
\end{aligned}
$$

Besides, $\left(\hat{x}^{k}, \hat{y}^{k}\right)$ is obtained location result by using No.k location algorithm, so the ultimate location coordinate of mobile station $(\hat{x}, \hat{y})$ is:

$$
\left[\begin{array}{l}
\hat{x} \\
\hat{y}
\end{array}\right]=\left[\begin{array}{l}
\frac{1}{\sum_{k=1}^{K} R_{k}^{-1}}\left(\sum_{k=1}^{K} \hat{x}^{k} R_{k}^{-1}\right) \\
\frac{1}{\sum_{k=1}^{K} R_{k}^{-1}}\left(\sum_{k=1}^{K} \hat{y}^{k} R_{k}^{-1}\right)
\end{array}\right]
$$

In the structure of GA algorithm, we can get the optimization equation as the following equation (6):

$$
h_{j}=\exp \left(-\frac{\left\|X-C_{j}\right\|}{2 b_{j}^{2}}\right), \quad j=1,2, \ldots, m(6)
$$

The output of the network is given as:

$$
y_{m}(k)=w h=w_{1} h_{1}+w_{2} h_{2}+\ldots+w_{m} h_{m}
$$

Assuming the ideal output is $y(\mathrm{k})$, the performance index function is:

$$
E(k)=\frac{1}{2}\left(y(k)-y_{m}(k)\right)^{2}
$$

The equation of basic function is as equation (9) as follows:

$$
\partial_{j}\left(C_{i j k l} \partial_{k} u_{l}+e_{k i j} \partial_{k} \varphi\right)-\rho \ddot{u}_{i}=0
$$

Under the linear relationship, basic equation is shown in equation (2):

$$
\partial_{j}\left(e_{i j k l} \partial_{k} u_{l}-\eta_{k i j} \partial_{k} \varphi\right)=0
$$

The linear differential equation can be expressed into the following simplified forms:

$$
\begin{aligned}
& L(\nabla, \omega) f(x, \omega)=0, \\
& \quad L(\nabla, \omega)=T(\nabla)+\omega^{2} \rho \mathrm{J}
\end{aligned}
$$




\section{EXPERIMENT RESULT}

Fig.1 shows the RMSE graph with changing the noise coefficient when mobile station is at location A. We can find that the errors of three algorithms all increased with the increase of noise coefficient. The real coordinates of mobile station is adopted as the initial value during simulating the Taylor series expansion method in noise environment, there-fore, the precision of Taylor series expansion method is significantly higher than Chan algorithm. The modified algorithm presented in this paper can make the initial value which is used to substitute into Taylor series expansion method approach to the real coordinate values of mobile station for it eliminates the estimated values which are excessively deviated after using Chan algorithm, therefore, the error of the modified algorithm is smaller than Chan algorithm. Due to some deviations existing between the initial values adopted in modified algorithm and real values, its errors are larger than that of Taylor series expansion. When the mobile station is located in location $\mathrm{A}$, the increasing amount of base station make the results of Chan algorithm more precise, however, it has little effects on the error of Taylor series expansion. To the modified algorithm which is based on Chan and Taylor, for the increasing amount of base station decreases the error of Chan algorithm, and in-creases the precision of initial values to some extent, thus making the results of Taylor series expansion method more precise, the errors of weighted results became smaller.

Fig. 2 shows the RMSE graph with changing the noise coefficient when the mobile station is at location B. From the graph it could be seen that location variation has some effects on location precision. When the mobile station is at location $\mathrm{B}$, the increasing amount of base stations also improves the location precision of Taylor series expansion method. From Fig.1 and Fig.2, we can find that in noise environment, the proposed algorithm attains better performance than Chan algorithm at different locations.

Fig. 3 and Fig. 4 show the RMSE graphs with changing NLOS coefficient when mobile stations are at location A and $\mathrm{B}$, respectively. From these graphs we find that with the increase of NLOS coefficient, performance of the three algorithms all decrease. In NLOS environment, the location precision of Taylor series expansion method is obviously better than Chan algorithm, and the location variation of mobile station also effects on location results. Because of integrating the advantages of Chan algorithm and Taylor series expansion method, the performance of our proposed algorithm is also better than Chan algorithm in NLOS environment.

\section{DISCUSSION}

The wireless multipath fading channel will greatly influence the location accuracy of any location measurement system. Major factors influencing multipath fading include multipath propagation, speed of the receiver, speed of the surrounding objects, and the transmission signal bandwidth. Multipath propagation refers to the fact that a signal transmitted from the sender can follow a multiple number of propagation paths to the receiving antenna. In our system, the performance is not affected by the speed of the receivers since all sensors and beacon nodes are stationary. However, a moving object in the surrounding area can cause interference. Our scheme mitigates the effect of multipath fading by measuring TDoA over mul-

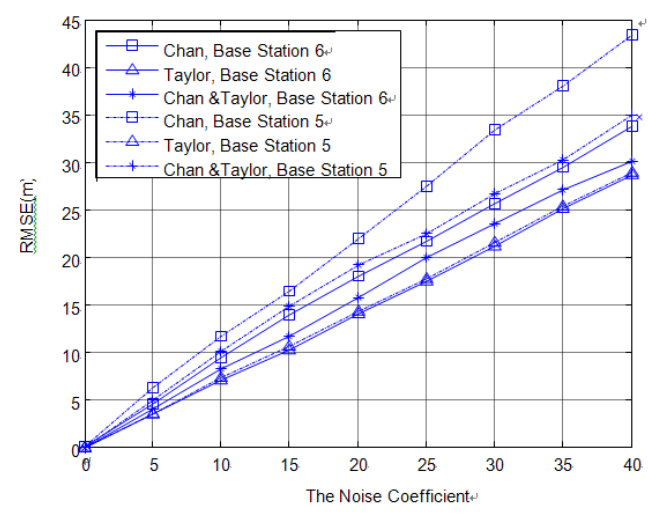

Figure 1. RMSE Graph with Changing the Noise Coefficient When Mobile Station Is at Location A

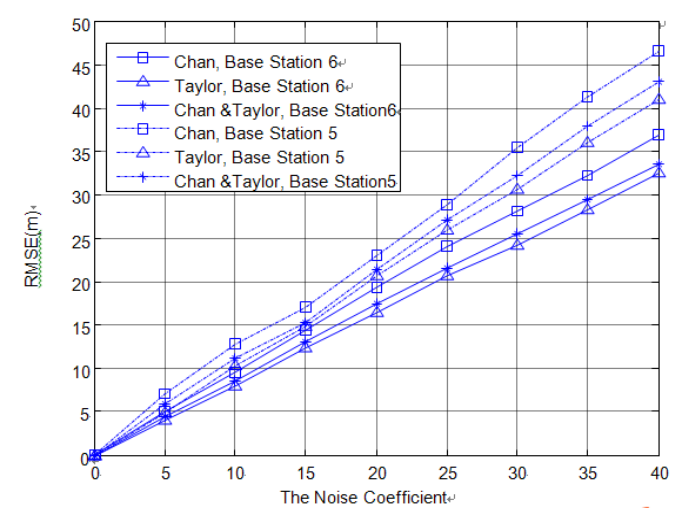

Figure 2. RMSE Graph with Changing the Noise Coefficient When Mobile Station Is at Location B

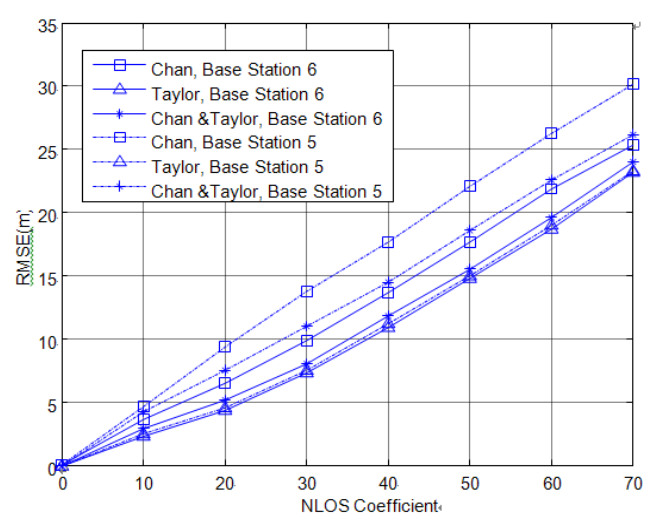

Figure 3. RMSE Graph with Changing NLOS Coefficient When Mobile Station Is at Location A

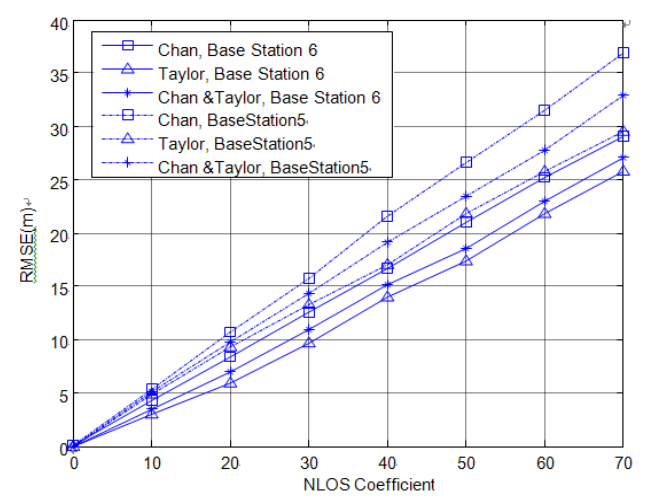

Figure 4. RMSE Graph with Changing NLOS Coefficient When Mobile Station Is at Location B 
tiple beacon intervals. TDoA measurements have been very effective in fading channels, as many detrimental effects caused by multipath fading can be cancelled.

Another factor related to wireless channels that causes location measurement errors is NLoS transmission. To mitigate NLoS effects, beacon nodes can be placed well above the surrounding objects such that there are line-ofsight transmission paths from beacon nodes to sensors.

In fact, during the experiment can be found, the received signal strength has a very distinct time-varying. Corresponding to different distances, the change of its location error is a random process. Figure 3-4 shows that, corresponding to different distances, by the line of sight distance of the normal number of errors estimated from the empirical model and the actual distance, i.e. ranging error. As can be seen from the figure, when the distance increases to a certain extent, the ranging error has increased significantly. And in the short distance (about 10 $m$ in the present experiment), the vast majority of the error is maintained at about $10 \%$. Description In the case of sight distance of close range accuracy is more reliable.

\section{CONCLUSION}

This paper introduces a new TDOA-based localization algorithm (TBL) for the large-scale, high-density wireless sensor networks which are aimed at real-time surveillance and unexpected incidents management. In particular, several means to improve the accuracy of distance measurement are investigated, and the TDOA method based on the sound wave and electromagnetic wave to locate in the large-scale WSN is discussed. Also, the well-designed circular location process has the advantage of better positioning accuracy and coverage percentage. This paper presents a three-dimensional joint wireless sensor networks localization algorithm based on wave interference sensor angle measurement (AOA) and time difference of arrival (TDOA). The algorithm has a high precision, can be effectively estimated three-dimensional space position sensor nodes. The algorithm is divided into three steps: Firstly, use the measurement algorithm based on wave interference angle sensor to calculate the angle of beacon nodes and unknown nodes; then using the measured angle and distance, using the proposed derived formula to calculate the precise coordinates of the unknown node.

\section{REFERENCES}

[1] P. J. Voltz, and D. Hernandez, "Maximum Likelihood Time of Arrival Estimation for Real-Time Physical Location Tracking of 802.11a/g Mobile Stations in Indoor Environments," IEEE Position Location and Navigation Symposium, pp:585-591,2014.

[2] H. Jing, "Routing optimization algorithm based on nodes density and energy consumption of wireless sensor network," Journal of Computational Information Systems, vol. 11, no.14, pp. 50475054, July2015.

[3] J. He and Y. Chen, "Remote Monitoring System of an Agricultural Tillage Machine Based on an Embedded ARM Technology Wireless Sensor," International Journal of Online Engineering, vol. 12, no 05, pp. 33-37, May 2016. https://doi.org/10.3991/ijoe. v12i05.5732

[4] T. J. S. Khanzada, A. R. Ali, and A. S. Omar, "Time Difference of Arrival Estimation using Super Resolution Algorithms to Minimize Distance Measurement Error for Indoor Positioning Systems," IEEE International Multi topic Conference (INMIC), pp. 443-447, 2008. https://doi.org/10.1109/inmic.2008.4777779

[5] P. C. Chen, "A Non-Line-of-Sight Error Mitigation Algorithm in Location Estimation," IEEE Wireless Communication and Networking Conference (WCNC'99), Vol. 1, USA, pp. 316-320, 1999.

[6] F. Zhao, G. Li, R. Zhang, et al., "Swarm-based intelligent optimization approach for layout problem," Multimedia Tools and Applications, pp. 1-17, 2015. https://doi.org/10.1007/s11042-015-3174$\underline{4}$

[7] A. Pisano, F. Bignami, and R. Santoleri. "Oil Spill Detection in Glint-Contaminated Near-Infrared MODIS Imagery," Remote Sensing, vol. 7, no.1, pp. 1112-1134, 2015. https://doi.org/10.3390/rs70101112

[8] H. Jing, "Node deployment algorithm based on perception model of wireless sensor network." International Journal of Automation Technology,vol.9, no.3, pp. 210-215, April 2015. https://doi.org/10.20965/ijat.2015.p0210

[9] Y. Li, Y. Zhang, J. Chen, et al. "Improved Compact Polarimetric SAR Quad-Pol Reconstruction Algorithm for Oil Spill Detection," IEEE Geoscience \& Remote Sensing Letters, vol. 7, no.1, pp. 1139-1142, 2014. https://doi.org/10.1109/LGRS.2013.2288336

\section{AUTHOR}

Ji Songbo is with Inner Mongolia University of Technology, College of Information Engineering, Hohhot 010080, China (jisongbo2008@126.com).

Submitted 09 September 2016. Published as resubmitted by the author 25 October 2016. 